\title{
Plan de evacuación de la unidad de cuidados intensivos: ¿un nuevo indicador de calidad?
}

\author{
M. Sánchez-Palacios ${ }^{a}$, R. Lorenzo Torrent ${ }^{a}$, L. Santana-Cabrera ${ }^{a}$,*, J.A. Martín García ${ }^{a}$, \\ S.G. Campos ${ }^{\mathrm{a}}$, V. Carrasco de Miguel ${ }^{\mathrm{b}}$ y Grupo de Trabajo del Plan de Autoprotección para \\ el Servicio de Medicina Intensiva
}

\author{
${ }^{a}$ Servicio de Medicina Intensiva, Hospital Universitario Insular de Gran Canaria, Las Palmas de Gran Canaria, \\ Islas Canarias, España \\ 'Unidad de Seguridad Hospitalaria, Hospital Universitario Insular de Gran Canaria, Las Palmas de Gran Canaria, \\ Islas Canarias, España
}

Recibido el 2 de marzo de 2009; aceptado el 6 de mayo de 2009

Disponible en Internet el 24 de septiembre de 2009

\section{PALABRAS CLAVE \\ Plan de catástrofe; \\ Triaje; \\ Unidad de cuidados \\ intensivos; \\ Sistemas de extinción \\ de incendios; \\ Cuidados de salud; \\ Indicadores de calidad}

\section{KEYWORDS}

Disaster planning;

Triage;

Intensive care units;

Fire extinguishing systems;

\begin{abstract}
Resumen
Las unidades de cuidados intensivos deben estar preparadas para un eventual desastre, bien sea de índole interno o externo, donde sea necesario evacuar a los pacientes ingresados. Deben disponer de un Plan de Emergencia y Autoprotección donde estén recogidos los criterios de evacuación de los pacientes, que debe conocer todo el personal que trabaja en el servicio. Para esto, habrá que hacer un triaje a los pacientes basándose en las prioridades de atención y según las posibilidades de supervivencia. El disponer de un plan de evacuación, conocido por todo el personal y actualizado mediante la realización de simulacros periódicos, debería incluirse como un indicador de calidad que se debería cumplir, ya que con esto se conseguiría una mejor atención al paciente en caso de presentarse una situación de desastre donde hubiere que evacuar la unidad de cuidados intensivos.

(c) 2009 Elsevier España, S.L. y SEMICYUC. Todos los derechos reservados.
\end{abstract}

Evacuation plan of an intensive care unit: A new quality indicator?

Abstract

The intensive care units must be prepared for a possible disaster, whether internal or external, in case it becomes necessary to evacuate the in-patients. They must have an Emergency and Self-protection Plan that includes the patient evacuation criteria and this

\footnotetext{
*Autor para correspondencia.

Correo electrónico: Isancabx@gobiernodecanarias.org (L. Santana-Cabrera).

- Formado por: Ana Casamitjana Ortega, Ángel Villanueva Ortiz, Mercedes Martín Fuentes, Ruperto Vega García, Marianela Quesada Vera, Josefa Torres Estupiñán y Rosa María Martín Monzón.
} 
Health care;

Quality indicators must be known by all the personnel who work in the service. For that reason, the patients must be triaged, based on their attention priorities, according to their survival possibilities. Having an evacuation, known by all the personnel and updated by means of the performance of periodic drills, should be included as a quality indicator that must be met, since this would achieve better attention to the patient in case of a disaster situation requiring the evacuation of the ICU.

(c) 2009 Elsevier España, S.L. and SEMICYUC. All rights reserved.

\section{Introducción}

Tras los atentados del 11 de septiembre de 2001 y de los desastres naturales, como el originado por el huracán Katrina o por terremotos (como el ocurrido en Turquía en 1999), cada vez se publican más artículos relacionados con la preparación y la actuación en los hospitales ante una emergencia a gran escala ${ }^{1-4}$.

Sin embargo, no existen muchos artículos en la literatura médica que traten sobre los planes de evacuación de los pacientes $\operatorname{críticos}^{5-8}$. Desde la perspectiva de los pacientes ingresados en la unidad de cuidados intensivos ( $\mathrm{UCl}$ ), cada unidad debería estar preparada para un eventual desastre, bien sea de índole interno o externo, donde sea necesario evacuar a los pacientes ingresados. La evacuación total de un hospital es, además de difícil, improbable, pero no así las evacuaciones parciales $o$ traslados a otras zonas no afectadas dentro del mismo hospital. Para esto, deben disponer de un Plan de Emergencia y Autoprotección donde estén recogidos los criterios de evacuación de los pacientes, que debe conocer todo el personal que trabaja en el servicio; a tal fin, es fundamental la realización de simulacros periódicos de entrenamiento.

\section{Desarrollo de nuestro Plan de Evacuación}

Tratamos de describir el trabajo realizado en relación con el diseño de un Plan de Evacuación en nuestra $\mathrm{UCI}$, que se encuentra en un hospital terciario con una capacidad de 425 camas, de 11 pisos de altura, construido con una disposición en 3 edificios, unidos por puentes (alas norte, sur y oeste). La UCI se encuentra en la tercera planta del recinto, en el ala sur, con una capacidad de 24 camas polivalentes (médico-quirúrgica y coronaria), dividida en 3 módulos de 8 camas, dentro de unas instalaciones modernas, inauguradas en el año 2001.

En ese mismo año se llevó a cabo en el Complejo Hospitalario la formación del Grupo de Gestión de Crisis, que constituyó posteriormente el Comité de Catástrofes, cuyas funciones son las de vigilar que se cumplan las condiciones de máxima seguridad física en el recinto sanitario y garantizar la seguridad de los pacientes, familiares y trabajadores mediante la elaboración, implantación y control del cumplimiento de los Planes de Emergencia y Autoprotección.

El Comité de Catástrofe, formado por un intensivista, una enfermera, un médico de urgencias, un médico de hospitalización, un responsable de la Unidad de Gestión de Calidad y el jefe de sección de la Unidad de Seguridad
Hospitalaria, fue el encargado de la realización del Plan de Emergencia y Autoprotección.

Como la $\mathrm{UCl}$ es un área particular respecto a otras áreas hospitalarias, se formó un grupo de trabajo que evaluó las características arquitectónicas y la particularidad y la complejidad del protocolo terapéutico del paciente crítico. Médicos, enfermeras y auxiliares clínicos pertenecientes al Servicio de Medicina Intensiva, con el asesoramiento del Comité de Catástrofes, formaron este grupo.

Tras diversas reuniones de trabajo se extrajeron las siguientes conclusiones. En primer lugar, se propuso un sistema de clasificación o triaje de los pacientes para ordenar la prioridad de evacuación. En segundo lugar, se valoró el sitio del hospital donde se los evacuaría; se buscó un refugio para los pacientes más graves en la misma planta con el objeto de realizar una evacuación horizontal en otra ala del hospital que reuniese las condiciones para acoger a los pacientes con necesidad de oxigenoterapia, ventilación mecánica y aparataje para soporte hemodinámico. El área elegida fue la del Hospital de Día de Oncología, en el ala oeste del hospital, a donde se evacuarían con el soporte vital necesario y existente en el servicio (respiradores, medicación, documentación médica, contenedor de emergencias, etc.). Los pacientes menos graves serían evacuados hacia el Área de Urgencias.

Se establecieron unos criterios de clasificación de los pacientes basados en 2 aspectos; por un lado, en relación con el esfuerzo terapéutico que deben recibir (tabla 1) y que se definieron sobre la base de una adaptación de las propuestas de Gómez Rubí en su libro “Ética en Medicina Crítica"9 y, por otro lado, por la situación clínica y la necesidad de soporte vital. Sobre la base de estos 2 factores se clasifican los pacientes en 4 grupos y se les asigna a cada uno un número, que va a indicar el orden de evacuación y el lugar a donde acudir. Estos criterios se evalúan diariamente mediante un programa informático que, en el momento de prescribir el tratamiento, con sólo introducir el grupo asistencial, las dosis de catecolaminas, la necesidad de oxígeno y de ventilación mecánica y el Coma Glasgow Score del enfermo, nos lo clasifica en el grupo de evacuación al que pertenece (tabla 2).

Los pacientes clasificados en el "grupo 1" se evacuarán en primer lugar hacia el Área de Urgencias del hospital y el personal del Servicio de Urgencias atenderá a este grupo.

Los clasificados en el "grupo 2" se evacuarán en segundo lugar, lo harán también hacia el Área del Hospital de Día de Oncología y el personal del Servicio de Medicina Intensiva atenderá a este grupo.

Los pacientes del "grupo 3" se evacuarán en tercer lugar, también hacia el Área del Hospital de Día de Oncología, en la misma planta, y el personal del Servicio de Medicina Intensiva atenderá a este grupo. 
Tabla 1 Clasificación de grupos asistenciales

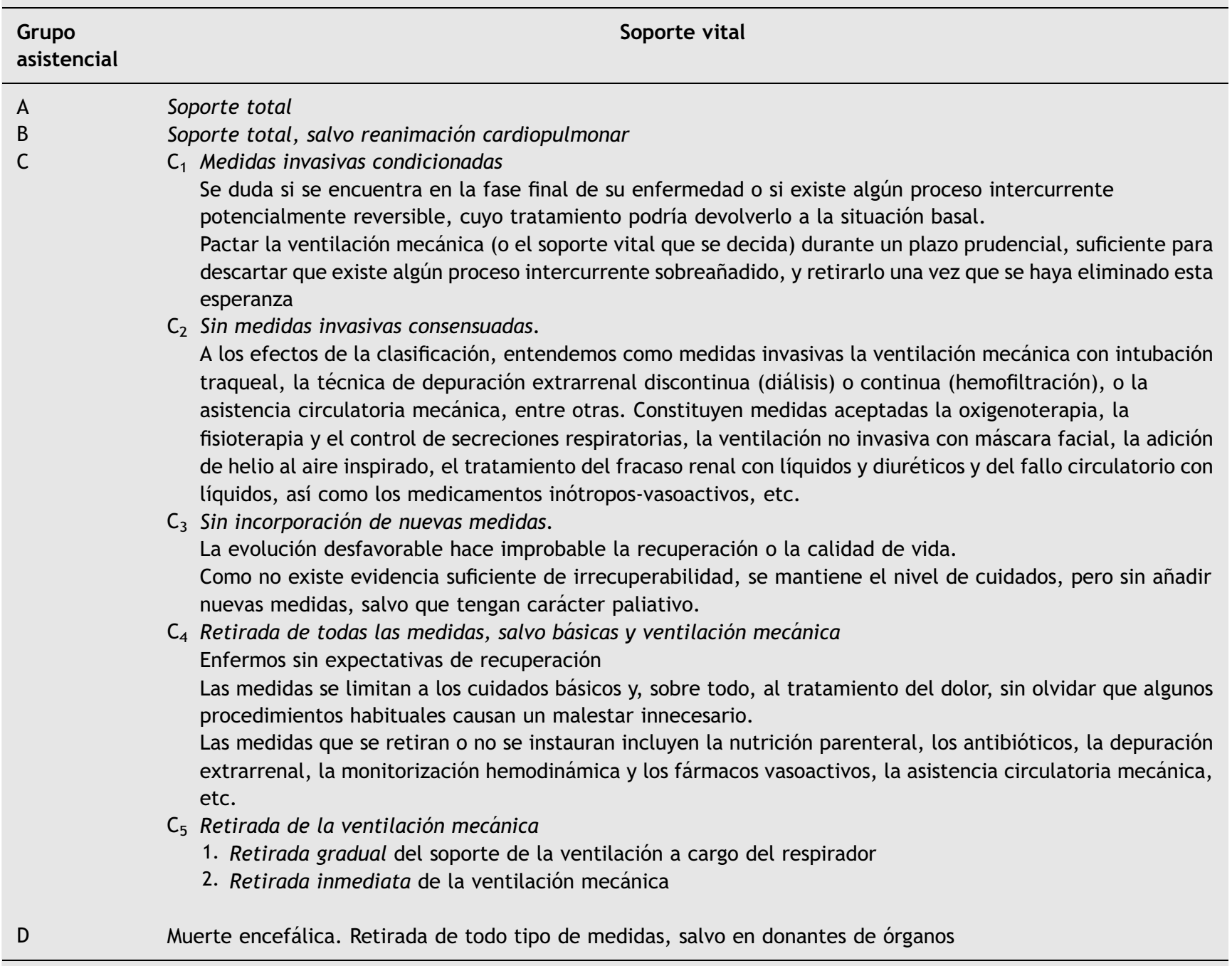

Finalmente, los pertenecientes al "grupo 4" se evacuarán en último lugar hacia el Área del Hospital de Día de Oncología, el personal del Servicio de Medicina Intensiva también atenderá a este grupo.

El Comité de Catástrofes, en el empeño de la divulgación de los planes de emergencia, diseñó unos programas de formación para el personal, cuyos objetivos eran el de informar sobre el Plan de Emergencias y Autoprotección, la formación básica en protección contra incendios y prácticas con el manejo del extintor con fuego real. También se elaboraron unas normas para garantizar una evacuación lo más ordenada y segura posible (tabla 3 ).

Asimismo, se llevó a cabo un simulacro de incendio en un módulo de la $\mathrm{UCl}$ con actores que simulaban los pacientes ingresados en ese momento, se realizó la evacuación y se siguió el orden de prioridad establecido, según el grupo al que pertenecían, hacia las zonas preestablecidas.

\section{Discusión}

En noviembre de 1995 se publicó en España, en el Boletín Oficial del Estado (BOE) español, la normativa sobre la prevención de riesgos laborales en la Ley $31 / 1995$, del 8 de noviembre, actualizado el 15 de diciembre de 2003, que tiene por objeto promover la seguridad y la salud de los trabajadores mediante la aplicación de medidas y el desarrollo de las actividades necesarias para la prevención de riesgos derivados del trabajo.

El 23 de marzo de 2007 se publicó en el BOE el Real Decreto 393/2007, por el que se aprueba la “Norma Básica de Autoprotección de los centros, establecimientos y dependencias dedicados a actividades que pueden dar origen a situaciones de emergencia", lo que constituye el marco legal que garantiza para todos los ciudadanos unos niveles adecuados de seguridad, eficacia y coordinación administrativa en materia de prevención y control de riesgos. Es de obligado cumplimiento para los establecimientos de usos sanitarios en los que se prestan cuidados médicos en régimen de hospitalización o de tratamiento intensivo o quirúrgico, con una disponibilidad igual o superior a 200 camas. Establece la obligación de elaborar, implantar y mantener operativos los planes de autoprotección, incidiendo no sólo en las actuaciones ante dichas situaciones, sino también y con carácter previo, en el análisis y la evaluación de los riesgos, en la adopción de 
Tabla 2 Clasificación del orden de evacuación

\begin{tabular}{|c|c|}
\hline 1. ${ }^{e r}$ lugar & $\begin{array}{l}\text { - Grupo asistencial A } \\
\text { - Conscientes }\end{array}$ \\
\hline Grupo 1 & $\begin{array}{l}\text { - No precisan ventilación mecánica. } \\
\text { - No precisan medicación vasoactiva. }\end{array}$ \\
\hline 2. ${ }^{\circ}$ lugar & $\begin{array}{l}\text { - Grupo asistencial A } \\
\text { - Conscientes }\end{array}$ \\
\hline Grupo 2 & $\begin{array}{l}\text { - Precisan de oxigenoterapia en mascarilla. } \\
\text { - Precisan de ventilación mecánica, invasiva o no, pero con } \mathrm{FiO}_{2}<50 \% \text { y PEEP }<7 \mathrm{~cm} \mathrm{H}_{2} \mathrm{O} \text {. } \\
\text { - Precisan catecolaminas (adrenalina o noradrenalina }<0,5 \mathrm{mcgr} / \mathrm{kg} / \mathrm{min} \text { ). }\end{array}$ \\
\hline 3. ${ }^{e r}$ lugar & $\begin{array}{l}\text { - Grupos asistenciales } B, C_{1} \circ C_{2} \\
\text { - No están conscientes: }\end{array}$ \\
\hline Grupo 3 & $\begin{array}{l}\text { - porque precisan sedación o } \\
\text { Precisan ventilación mecánica con oxígeno a altas concentraciones }(50-80 \%) \text { y PEEP elevada }\left(>8-10 \mathrm{~cm} \mathrm{H}_{2} \mathrm{O} \text { ). }\right. \\
\text { - Precisan catecolaminas en dosis elevadas (adrenalina o noradrenalina 0,5-1 } \mathrm{mcgr} / \mathrm{kg} / \mathrm{min}) \text {, con una índice } \\
\text { cardíaco }>1,8 \mathrm{l} / \mathrm{min} / \mathrm{m}^{2} \text { o necesidad de contrapulsación aórtica. }\end{array}$ \\
\hline $4 .^{\circ}$ lugar & $\begin{array}{l}\text { - Grupos asistenciales } C_{3}, C_{4}, C_{5} \circ D \\
\text { - No conscientes: con daño neurológico grave (CGS } \leq 5 \text { puntos) }\end{array}$ \\
\hline Grupo 4 & $\begin{array}{l}\text { - Fallo respiratorio grave, con ventilación mecánica, que requiere concentraciones muy elevadas de oxígeno } \\
(\geq 80 \%) \text { y PEEP }>10 \\
\text { - Precisan dosis de catecolaminas muy elevadas (adrenalina o noradrenalina }>1 \mathrm{mcgr} / \mathrm{kg} / \mathrm{min} \text { ). }\end{array}$ \\
\hline
\end{tabular}

CGS: Coma Glasgow Score; $\mathrm{FiO}_{2}$ : fracción inspirada de oxígeno; PEEP: presión espiratoria al final de la espiración.

Tabla 3 Sistemática de evacuación

- Mantener la calma, no fomentar situaciones alarmistas

- Evacuar con rapidez, sin gritos ni aglomeraciones, guiando y prestando ayuda a los ocupantes hacia las vías de evacuación

- No llevar consigo objetos personales

- No intentar recuperar ningún objeto que se caiga

- Promover la ayuda mutua

- No volver a entrar en el área evacuada

- Cerrar las puertas y las ventanas que se encuentren en el camino de la evacuación

- Acompañar al personal evacuado al punto de reunión correspondiente

- Mantener el orden y la tranquilidad en las áreas de seguridad

- Una vez finalizada la evacuación, comprobar que se ha efectuado completamente verificando que no haya ausencias

- Mantener libre la línea telefónica y comunicar que la planta ha sido evacuada

- Cuando la planta esté evacuada, se deberá situar una persona en la salida a fin de impedir el paso a personas que no pertenezcan a los equipos de emergencia.

medidas preventivas y de control de los riesgos, así como en la integración de las actuaciones en emergencia en los correspondientes Planes de Emergencia de Protección Civil.

Si bien ante una catástrofe parece a priori que un hospital no puede ser evacuable, lo cierto es que puede llegar a necesitarse, aunque sea como última medida. La razón argumentada de la dificultad en la evacuación no debe utilizarse para no planificarla, ya que pese a su dificultad y a sus consecuencias, puede evitarse que la catástrofe sea mayor.

$\mathrm{Si}$ se desea que la evacuación sea verdaderamente eficaz, hay que partir de 2 premisas fundamentales: una, que sea ordenada y otra, que se sigan normas preestablecidas. Esto viene determinado por el escaso o limitado tiempo con el que se cuenta para evacuar a los pacientes y por la necesidad de solventar las reacciones de pánico que suele provocar cualquier siniestro. Además, cualquier desastre que precise de la evacuación de una $\mathrm{UCI}$ no necesariamente ocurrirá en el momento donde existe más personal, así que es de vital importancia que el personal que atiende diariamente al enfermo crítico, sobre todo de enfermería, conozca las normas básicas de evacuación ${ }^{1,2,10}$. 
Por tanto, toda sistemática de evacuación debe constar de unas normas generales para el personal, una definición de prioridades en función del área por evacuar, el tipo de enfermos y la disponibilidad de personal y, por último, de una metodología del traslado de enfermos teniendo en cuenta las características de éstos.

El triaje es un método que ha utilizado clásicamente la medicina de emergencias para la selección y la clasificación de los pacientes basándose en las prioridades de atención según las posibilidades de supervivencia. Cualquier sistema de triaje se basa en una escala de priorización útil, válida y reproducible; así, se han desarrollado diversos sistemas o modelos de triaje. La lección mejor aprendida tras el atentado de Nueva York se basa en que todo su sistema de emergencia sanitaria habla el mismo lenguaje, utiliza el índice START para clasificar a las víctimas, que etiquetan con el Sistema Mettag ${ }^{11}$.

En una evacuación, el sistema de triaje trata de priorizar el traslado de aquellos pacientes con más posibilidades de supervivencia, al precisar de un menor apoyo de soporte vital $^{12}$. Es importante realizar este triaje previamente a la aparición de una situación de catástrofe, por lo que nosotros recomendamos que se haga diariamente, al incluir tanto el soporte vital que está precisando en ese momento como el grupo asistencial en que se encuentra, y éstos deben asignarse en condiciones normales de atención médica y no en situaciones de emergencia. La limitación del esfuerzo terapéutico incluida en los diversos grupos asistenciales se realiza con el fin de evitar el encarnizamiento terapéutico, debe fundarse en la medicina basada en la evidencia, debe tener en cuenta la voluntad del enfermo (instrucciones previas), debe tomarse en consenso del equipo asistencial y deben tener conocimiento de ésta sus familiares ${ }^{13,14}$.

La evacuación del paciente crítico debe estar protocolizada, debe disponer de un médico, una enfermera, monitorización del paciente, disponibilidad de medicación de emergencia y de un desfibrilador, si es posible, y mover al paciente en su propia cama. Todo esto ante una situación de improvisación, que probablemente sea algo que no se vaya a realizar. La evacuación ante un desastre interno, como por ejemplo un incendio, ha de ser siempre que sea posible en horizontal, es decir, hacia otras zonas del hospital, en la misma planta del edificio, donde se pueda estar aislado del fuego. El problema se complica cuando se precisa una evacuación vertical, es decir hacia plantas inferiores ${ }^{7,15}$, sobre todo para tener en cuenta en las $\mathrm{UCl}$ que se encuentran en pisos altos del recinto hospitalario, donde los recursos humanos y materiales que se utilizan para trasladar a los enfermos críticos se multiplican.

La Sociedad Española de Medicina Intensiva Crítica y Unidades Coronarias ha elaborado unos indicadores de calidad en el enfermo crítico $^{16,17}$ entre los que se incluyen algunos indicadores de estructura, tales como la presencia de un intensivista las $24 \mathrm{~h}$ del día, la existencia de protocolos básicos o de un equipo de emergencias médicas. Nosotros pensamos que el poseer un Plan de Emergencia y Autoprotección, conocido por todo el personal y actualizado, debería incluirse como un indicador de calidad por cumplir, ya que con esto se conseguiría una mejor atención al paciente en caso de presentarse una situación de desastre donde hubiere que evacuar la UCI.

Además, hay que pensar también que ante la posibilidad de que ocurra un desastre de tal magnitud que obligue a desalojar la $\mathrm{UCI}$, es probable que pueda haber heridos graves que también debamos atender y para lo que tenemos también que estar preparados ${ }^{18}$.

En conclusión, la disponibilidad de un Plan de Emergencia y Autoprotección en la UCl que permita llevar a cabo la evacuación de los pacientes mediante un triaje estructurado, realizado previamente a la aparición de la catástrofe, es una necesidad ineludible dentro de un sistema sanitario de calidad, cuya eficacia debería validarse y confirmarse periódicamente mediante la realización de simulacros.

\section{Bibliografía}

1. Orlando S, Bernard ML, Mathews P. Neonatal nursing care issues following a natural disaster: Lessons learned from the Katrina experience. J Perinat Neonatal Nurs. 2008;22:147-53.

2. Bernard M, Mathews PR. Evacuation of a maternal-newborn area during hurricane Katrina. MCN Am J Matern Child Nurs. 2008;33:213-23.

3. Brevard SB, Weintraub SL, Aiken JB, Halton EB, Duchesne JC, McSwain Jr NE, et al. Analysis of disaster response plans and the aftermath of hurricane Katrina: Lessons learned from a level I trauma center. J Trauma. 2008;65:1126-32.

4. Halpern P, Rosen B, Carasso S, Sorkine P, Wolf Y, Benedek P, et al. Intensive care in a field hospital in an urban disaster area: Lessons from the August 1999 earthquake in Turkey. Crit Care Med. 2003;31:1410-14.

5. Carey MG. Smoked out: Emergency evacuation of an ICU. Am J Nurs. 2007;107:54-7.

6. Cybulski P. Evacuation of a critical care unit. Dynamics. 2003 Fall;14:21-3.

7. Manion P, Golden IJ. Vertical evacuation drill of an intensive care unit: Design, implementation, and evaluation. Disaster Manag Response. 2004;2:14-19.

8. Schultz R, Pouletsos C, Combs A. Considerations for emergencies \& disasters in the neonatal intensive care unit. MCN Am J Matern Child Nurs. 2008;33:204-10 quiz 211-2.

9. Gómez Rubí JA. Ética en Medicina Crítica. Triacastela. 2002.

10. Castle NG. Nursing home evacuation plans. Am J Public Health. 2008;98:1235-40.

11. Cook L. The World Trade Center attack. The paramedic response: An insider's view. Crit Care. 2001;5:301-3.

12. Consensus statement on the triage of the critically ill patients: Society of Critical Care Medicine Ethics Committee. JAMA. 1994;271:1200-3.

13. Cabré L, Solsona JF, Grupo de Trabajo de Bioética de la SEMICYUC. Limitación del esfuerzo terapéutico en Medicina Intensiva. Med Intensiva. 2002;26:304-11.

14. Monzón Marín JL, Saralegui Reta A, Abizanda i Campos R, Cabré Pericas L, Iribarren Diarasarri S, Martín Delgado MC, et al. Recomendaciones de tratamiento al final de la vida del paciente crítico. Med Intensiva. 2008;32:121-33.

15. Gildea JR, Etengoff S. Vertical evacuation simulation of critically ill patients in a hospital. Prehosp Disaster Med. 2005;20:243-8.

16. Sociedad Española de Medicina Intensiva Crítica y Unidades Coronarias. Indicadores de calidad en el enfermo crítico. Madrid; 2005. [citado 24 Ene 2009] Disponible en: URL: http://www.calidad.semicyuc.org.

17. Martín MC, Cabré L, Ruiz J, Blanch L, Blanco J, Castillo F, et al. Grupos de Trabajo de la Sociedad Española de Medicina Intensiva Crítica y Unidades Coronarias (SEMICYUC), Sociedad Española de Enfermería Intensiva y Unidades Coronarias (SEEIUC) y Fundación AVEDIS Donabedian (FAD). Indicadores de calidad en el paciente crítico. Med Intensiva. 2008;32:23-32.

18. Roccaforte JD, Cushman JG. Disaster preparation and management for the intensive care unit. Curr Opin Crit Care. 2002;8:607-15. 